\title{
Imaging Cerebral Gene Transcripts in Live Animals
}

\author{
Christina H. Liu, ${ }^{1,2,3}$ Young R. Kim, ${ }^{1,3}$ Jia Q. Ren, ${ }^{1,3}$ Florian Eichler, ${ }^{4}$ Bruce R. Rosen, ${ }^{1,3}$ and Philip K. Liu ${ }^{2,3}$ \\ ${ }^{1}$ Athinoula A. Martinos Center for Biomedical Imaging, ${ }^{2}$ Transcript Imaging and NeuroRepair Laboratory, ${ }^{3}$ Department of Radiology, and ${ }^{4}$ Department of \\ Neurology, Massachusetts General Hospital, Charlestown, Massachusetts 02129
}

To circumvent the limitations of using postmortem brain in molecular assays, we used avidin- biotin binding to couple superparamagnetic iron oxide nanoparticles (SPIONs) $(15-20 \mathrm{~nm}$ ) to phosphorothioate-modified oligodeoxynucleotides (sODNs) with sequence complementary to $c$-fos and $\beta$-actin mRNA (SPION-cfos and SPION- $\beta$ actin, respectively) (14-22 nm). The Stern-Volmer constant for the complex of SPION and fluorescein isothiocyanate (FITC)-sODN is $3.1 \times 10^{6} / \mathrm{m}$. We studied the feasibility of using the conjugates for in vivo magnetic resonance imaging (MRI) to monitor gene transcription, and demonstrated that these complexes at $40 \mu \mathrm{g}$ of Fe per kilogram of body weight were retained at least $1 \mathrm{~d}$ after intracerebroventricular infusion into the left ventricle of C57Black6 mice. SPION retention measured by MRI as $T_{2}{ }^{*}$ or $R_{2}{ }^{*}$ maps $\left(R_{2}{ }^{*}=1 / T_{2}{ }^{*}\right)$ was compared with histology of iron oxide (Prussian blue) and FITC-labeled sODN. We observed significant reduction in magnetic resonance $(\mathrm{MR}) \mathrm{T}_{2}{ }^{*}$ signal in the right cortex and striatum; retention of SPION-cfos and SPION- $\beta$ actin positively correlated with c-fos and $\beta$-actin mRNA maps obtained from in situ hybridization. Histological examination showed that intracellular iron oxide and FITC-sODN correlated positively with in vivo MR signal reduction. Furthermore, in animals that were administered SPION-cfos and amphetamine ( $4 \mathrm{mg} / \mathrm{kg}$, i.p.), retention was significantly elevated in the nucleus accumbens, striatum, and medial prefrontal cortex of the forebrain. Control groups that received SPION-cfos and saline or that received a SPION conjugate with a random-sequence probe and amphetamine showed no retention. These results demonstrated that SPION-sODN conjugates can detect active transcriptions of specific mRNA species in living animals with MRI.

Key words: amphetamine; antisense delivery; aptamer; drug addiction; gene transcription; immediate-early genes; nanotechnology; signal transduction

\section{Introduction}

Fos antigen, the product of c-fos mRNA, is one of the components for transcription factor activator protein-1 which has been shown to be a neuronal marker for stress, learning, and activation (Hope et al., 1992; Kaczmarek et al., 2002; Conversi et al., 2004; Gass et al., 2004; Raivich and Behrens, 2006), and controls the expression of several growth factors (Ricart et al., 2006). Amphetamine, a fat-soluble substance, reaches the brain quite easily after intraperitoneal injection and has been shown to elevate different members of the Fos peptide superfamily in the nucleus accumbens, cingulate and motor cortices, and striatum of rodent forebrain (Moratalla et al., 1996; Schmauss, 2000; Kiyatkin, 2004). Nonetheless, associations between neuronal activation and drug-related behavior are less well understood, partly because of difficulties in measuring less abundant levels of c-fos mRNA (Parelkar and Wang, 2004) and termination of animal subjects to obtain autopsy samples for assay. Availability of MRI for drug-induced gene transcription in live animals would overcome some of these difficulties.

Received July 31, 2006; revised Nov. 30, 2006; accepted Dec. 13, 2006.

This work was supported by National Institute of Neurological Disorders and Stroke Grant R01NS45845 and National Center for Research Resources Grant P41RR14075 and funds from the Mental Illness and Neuroscience Discovery Institute and the Athinoula A. Martinos Center for Biomedical Imaging. We thank Drs. Helen D'Arceuil, Joseph Mandeville, John Marota, and David Sosnovik for insightful discussion and Dr. C. Farrar, S. Nagpal, and John Moore for technical assistance.

Correspondence should be addressed to Dr. Philip K. Liu, Department of Radiology, Massachusetts General Hospital, 149 13th Street, Room 2410, Charlestown, MA 02129. E-mail: philipl@nmr.mgh.harvard.edu. DOI:10.1523/JNEUROSCI.4660-06.2007

Copyright $\odot 2007$ Society for Neuroscience $\quad$ 0270-6474/07/260713-10\$15.00/0
Recently, $\mathrm{T}_{1}$ - and $\mathrm{T}_{2}$-weighted high-resolution magnetic resonance (MR) techniques have been used to evaluate biological processes. Superparamagnetic iron oxide nanoparticles (SPIONs) ( $\mathrm{a} \mathrm{T}_{2}$ contrast agent) can produce significantly strong image contrast by reducing $\mathrm{T}_{2}$ relaxation time to generate localized signal reduction in $\mathrm{T}_{2}$-weighted $\mathrm{MR}$ images (VanLandingham et al., 1998). SPIONs have been shown to be well tolerated as blood-pool contrast agents, and are used in functional magnetic resonance imaging (MRI) studies in live animals, because $\mathrm{T}_{2}$ signal reduction reflects blood circulation in regions with high cellular activities (Mandeville et al., 1998, 2001). MR contrast agents have also been used to study axonal transport (Petropoulos et al., 1995), and to track transplanted cells in live animals using MRI (Bulte et al., 2002).

Single-stranded phosphorothioate-modified oligodeoxynucleotides (sODNs) with sequences complementary to mRNA have been shown to have specific targeting effects on cerebral mRNAs such as c-fos (Chiasson et al., 1992; Konradi et al., 1994; Liu et al., 1994; Morrow et al., 1999; M. Zhang et al., 1999; Y. Zhang et al., 1999; Tolliver et al., 2000). We have demonstrated sODN uptake in the cerebellum and olfactory bulb of the rat brain after intracerebroventricular infusion (supplemental Fig. S1 $A, B$, available at www.jneurosci.org as supplemental material) (Liu et al., 1994; Cui et al., 1999). To promote the development of ODN-based MRI contrast probes as aptamers for in vivo $\mathrm{MR}$ imaging and targeting of cerebral gene transcription, we developed a c-fos probe labeled with the reporter contrast SPION (SPION-cfos) for 
imaging the brains of live C57Black6 mice (see Fig. 1A). We propose that SPION retention reflects gene transcripts in brains of normal animals when reported by SPION-sODN probes with different target sequences (see Fig. $1 B$ ). Specifically, this report demonstrates (1) effective linkage between SPION and sODN via conjugation of avidin and biotin, (2) that the stability of SPION-cfos in mouse brain is sufficient for MR assessment, (3) positive correlation of cellular iron oxide and MR image intensity, and (4) that acute amphetamine administration induces specific elevated SPION retention in the forebrains of animals that received SPION-cfos.

\section{Materials and Methods}

Contrast probe preparation

SPION-NeutrAvidin. SPION was prepared and purified for these studies in the Athinoula A. Martinos Center for Biomedical Imaging at Massachusetts General Hospital (MGH), as described previously (Shen et al., 1993; Lind et al., 2002). Freshly synthesized SPION was functionalized with cyanogen bromide (Marshall and Rabinowitz, 1976), and linked to NeutrAvidin (NA) in the presence of $1 \mathrm{~m}$ sodium cyanoborohydride (both from Pierce Biotechnology, Rockford, IL). The resulting covalently linked product, SPION-NA, was filtered and dialyzed against a $20 \times$ volume of sodium citrate buffer solution ( $25 \mathrm{~mm}, \mathrm{pH} 8.0)$, using a Centricon Plus-100 filter (100 kDa cutoff; Millipore, Bedford, MA). Activated SPION (SPION-NA) was stored in an amber-colored bottle at $4^{\circ} \mathrm{C}$, at a concentration of $4 \mathrm{mg}$ of iron per milliliter of sodium citrate buffer. Iron concentrations in SPION samples were determined by optical absorbance at $410 \mathrm{~nm}$ after treatment with hydrogen peroxide $(0.03 \%)$ and $6 \mathrm{~N}$ hydrogen chloride (de Marco et al., 1998).

Conjugation of biotinylated sODN to SPION-NA. We synthesized three $5^{\prime}$-biotin-labeled antisense sODN: sODN-cfos, 5'-catcatggtcgtggtttgggcaaacc-3' (Liu et al., 1994; Y. Zhang et al., 1999); ODN- $\beta$ actin, 5' acgcagctcagtaacagtccgccta-3' (Schedlich et al., 1997); and sODN-Ran, a randomized sequence, as negative control, 5' -gggatcgttcagagtctag- $3^{\prime}$ (Zhang et al., 2001). All sODN were purified using PAGE. The sequence of sODN-cfos is complementary to rat c-fos mRNA; we used it because the sODN made with mouse sequence contains CCCC, which compromises its stability during storage (our unpublished observation). To directly observe the sODN, we also synthesized sODN with fluorescein isothiocyanate (FITC) on the $5^{\prime}$ terminus and biotin on the $3^{\prime}$ terminus (FITC-sODN-biotin). SPION-NA (250 nmol of Fe) was incubated with the biotinylated sODN (FITC-sODN-biotin) $(1 \mathrm{nmol})$ at room temperature for $30 \mathrm{~min}$ and the mixture was filter-dialyzed with three washes of sodium citrate buffer (25 mM, $\mathrm{pH}$ 8) in a centrifugal filter device (Microcon YM-30; Millipore). We incubated unconjugated SPION of the same lot from which SPION-sODN had been made with saline. The probes were resuspended in $36 \mu \mathrm{l}$ of sodium citrate buffer. After conjugation, the probes were stored at $4^{\circ} \mathrm{C}$ (no longer than $24 \mathrm{~h}$ ). Immediately before infusion, we added $4 \mu \mathrm{l}$ of lipofectin $(0.1 \mathrm{mg} / \mathrm{ml}$; Invitrogen, San Diego, CA).

Characterization of SPION-NA and SPION-sODN in vitro. The core sizes of the conjugates were measured using a Zetasizer particle characterization system (ZEN3600 with $4 \mathrm{~mW} 633 \mathrm{He}-\mathrm{Ne}$ laser; size range, 0.6 $\mathrm{nm}$ to $6 \mu \mathrm{m}$; Malvern Instruments, Southborough, MA). The volume fraction $(\phi)$ of nanoparticles was used to determine the number $(N)$ of iron atoms per SPION; the volume-weighted diameter of the SPIONcfos nanoparticle in a dilute solution was determined with a modified equation derived by Reynolds et al. (2005). The concentration of SPION was calculated by dividing the iron concentration by the number $(N)$ of iron atoms per nanoparticle.

The linkage of SPION and SODN using gel shift assay. The binding capacity for biotin in SPION-NA was determined in triplicate by incubating 100 pmol of FITC-sODN-biotin with 11-171 pmol of SPION-NA at room temperature for $1 \mathrm{~h}$, followed by resolution by electrophoresis in an agarose gel $(0.8$ or $3 \%)$. Because a magnet column cannot partition FITC-sODN-biotin and SPION-cfos, we used gel electrophoresis to separate bound and unbound FITC-sODN-biotin at room temperature. With a secondary absorption peak at $320 \mathrm{~nm}$, FITC-sODN-biotin can be visualized with a UV light source $(302 / 365 \mathrm{~nm})$. Images were captured using a digital camera with a FLSC-400 filter (Alpha Innotech, San Leandro, CA). Binding between FITC-sODN-biotin and SPION-NA will cause the former to migrate more slowly together with SPION and appear as a shift-up in the gel. The appearance of an excess unbound FITCsODN-biotin band after the saturation point was reached indicated binding capacity; the saturation concentration of FITC-sODN-biotin divided by SPION-NA concentration yielded the number of binding sites (to the nearest whole number). For binding site calculation, please see the example in the supplemental material (available at www.jneurosci.org).

To demonstrate linkage between SPION-NA and FITC-sODN-biotin, we excised four gel slices from each lane, placed each slice in an Eppendorf vial, and then melted and resolidified the gel to ensure uniform distribution of MR contrast agent. We used a 9.4 tesla MRI system to measure the $\mathrm{T}_{2}$ relaxation times of the gel slices.

Fluorescence emission and absorption spectra measurements. SternVolmer plots were determined in 25 mm sodium citrate buffer, $\mathrm{pH}$ 8.0, by mixing SPION-NA solution $(0-6 \mu \mathrm{M})$ with free and unbound FITCsODN-biotin solutions ( 5 or $10 \mu \mathrm{M}$ ), for solutions with final concentrations ranging from 60 to $900 \mathrm{~nm}$. Fluorescence emission for each solution was measured with a PerkinElmer (Wellesley, MA) Victor ${ }^{3}$ V 1420 Multilabel Counter (Wallac) at $450 \mathrm{~nm}$ excitation and $510 \mathrm{~nm}$ emission. Each concentration of SPION-NA with or without FITC-sODN-biotin was prepared in quadruplicate; two to three concentrations were measured at a time and repeated five times to generate a seven-point curve in the plot. Fluorescence in each concentration was averaged; data were analyzed in GraphPad Prism 4 (GraphPad Software, San Diego, CA). Absorption spectra of FITC-sODN-biotin and SPION-sODN-FITC were measured using a Shimadzu (Tokyo, Japan) UV2101 spectrophotometer; measurements were repeated in triplicate and determined to have two absorption peaks at $495 \mathrm{~nm}$ (primary) and $320 \mathrm{~nm}$ (secondary).

\section{Delivery of contrast conjugates}

All procedures and animal care practices adhered strictly to Association for the Assessment and Accreditation of Laboratory Animal Care, Society for Neuroscience, and institutional guidelines for experimental animal health, safety, and comfort. Male C57Black6 mice ( $24 \pm 3 \mathrm{~g}$; Taconic, Germantown, NY) were anesthetized with ketamine ( $42 \mathrm{mg} / \mathrm{kg}$, i.p. ) and xylazine ( $8 \mathrm{mg} / \mathrm{kg}$, i.p.), and the contrast conjugate was delivered to the brain at a dose of $1 \mu \mathrm{g}$ per mouse $(0.04 \mathrm{mg} / \mathrm{kg} \mathrm{Fe})$ via intracerebroventricular infusion. The dose administered to each mouse was lower than the nontoxic $1.1 \mathrm{mg} / \mathrm{kg}$ Fe dose used in humans (Saini et al., 2000). We used the minimum number of animals necessary, as calculated with a power calculation using the mean and SEM and in-house software by Wayne W. LaMorte (Boston University Medical Center, Boston, MA) ("Sample Size Calculations" available to all animal researchers at MGH). Unconjugated SPION or SPION-cfos in $2 \mu$ l of sodium citrate buffer was delivered $(0.2 \mu \mathrm{l}$ per minute) into the ventricle (stereotactic coordinates from bregma: left-right, $-1.0 \mathrm{~mm}$; anterior-posterior, $-0.4 \mathrm{~mm}$; dorsal-ventral, $-3.0 \mathrm{~mm}$ ) guided by a stereotactic device (Stoelting, Wood Dale, IL). Afterward, the burr hole in the skull was sealed with bone wax, and the incision was closed with sutures. The infusion dose of SPION was $5 \mu \mathrm{g}$ per mouse $(0.2 \mathrm{mg} / \mathrm{kg} \mathrm{Fe})$, because at low dose SPION is not retained in the brain for MR imaging.

To test SPION retention after amphetamine-induced neuronal stimulation, we injected amphetamine $(4 \mathrm{mg} / \mathrm{kg})$ or saline $(10 \mathrm{ml} / \mathrm{kg})$ intraperitoneally $4 \mathrm{~h}$ after SPION-sODN infusion; brain MRI was acquired $3 \mathrm{~h}$ later.

\section{Histology and molecular biological assay}

Postmortem tissue preparation. Animals were anesthetized (with ketamine and xylazine as for probe delivery) and the brains were removed from the animals' skulls and flash-frozen in an optimal cutting temperature compound (Sakura Finetek, Torrance, CA). For MR microscopy, mice were perfused transcardially with $15 \mathrm{ml}$ heparinized saline at a rate of $10 \mathrm{ml} / \mathrm{min}$, and then with $15 \mathrm{ml}$ of freshly prepared paraformaldehyde (PFA) (4\%) in $0.1 \mathrm{M}$ PBS, $\mathrm{pH} 7.4$, at a rate of $10 \mathrm{ml} / \mathrm{min}$. Once removed from the animals' skulls, the brains were stored in PFA solution at $4^{\circ} \mathrm{C}$ overnight, and then incubated for $16 \mathrm{~h}$ in $20 \%$ sucrose/PBS solution at $4^{\circ} \mathrm{C}$ to chase the PFA. Brains were either immediately scanned with high resolution MR microscopy in a 14 tesla MRI system or embedded in paraffin (Cui et al., 1999). 
Histological and immunohistochemical staining and molecular assay using in situ hybridization. Postmortem tissue samples were prepared $1 \mathrm{~d}$ after infusion, and coronal tissue slices $(20-100 \mathrm{~mm}$ thick) were prepared from paraffin-embedded blocks as described previously (Liu et al., 1994). A mercury light source, filtered with a $495 \mathrm{~nm}$ broad spectrum suitable for wavelengths of $470 \mathrm{~nm}$ (excitation) and $525 \mathrm{~nm}$ (emission), was used to observe SPION-sODN-FITC. We captured the digital image with a SPOT 1 digital camera (Diagnostic Instruments, Detroit, MI) mounted on an Olympus (Tokyo, Japan) BX-51 microscope (Cui et al., 1999). An adjacent tissue slice was stained with Prussian blue (PB) with $2 \%$ potassium ferrocyanide in $2 \% \mathrm{HCl}$ (Perl's method) and nuclear fast red (NFR) (Fisher Scientific, Houston, TX) counterstain to detect intracellular iron. For detection of mRNA transcription using in situ hybridization, all protocols including tissue section preparation, ${ }^{32} \mathrm{P}$-labeled RNA probe (antisense and sense orientation), and autoradiogram exposure were performed as described previously (Cui et al., 1999a).

\section{MRI protocol}

In vitro MRI of SPION probes. Calibration experiments were performed to investigate the $\mathrm{MRT}_{1}$ and $\mathrm{T}_{2}$ relaxivities ( $\mathrm{r} 1$ and $\mathrm{r} 2$, respectively) of the SPION conjugates used in this study, with different SPION concentrations in $25 \mathrm{~mm}$ sodium citrate buffer based phantoms at field strengths of 9.4 and 14 tesla, as described previously (Liu et al., 2004). The slopes of the linear fit are the relaxivities $(\mathrm{r} 1, \mathrm{r} 2)$ of each contrast probe.

In vivo data acquisition. In vivo image acquisition was performed using a 9.4 tesla MRI scanner (Bruker Avance system, Bruker Biospin MRI; Bruker, Billerica, MA) at $30 \mathrm{~min}, 7 \mathrm{~h}$, and $1 \mathrm{~d}$ after intracerebroventricular infusion of SPION. Animals were anesthetized with pure $\mathrm{O}_{2}$ and $2 \%$ halothane $(800 \mathrm{ml} / \mathrm{min}$ flow rate) for all time points except that immediately after the intracerebroventricular infusion procedure. We positioned a custom-built $1 \mathrm{~cm}$ transmit/receive surface coil on the heads of the animals, which were placed in the prone position in a home-built cradle. Gradient echo (GE) images of constant repetition time (TR) and incremental echo spacing (TE) were acquired at each time point along the axial direction. Acquisition parameters were as follows: TR, $500 \mathrm{~ms}$; TE, 3, 4, 6, 8, and $10 \mathrm{~ms}$; flip angle, 30; $200.5 \mathrm{~mm}$ slices; $15 \times 15 \mathrm{~mm}$ field of view; $128 \times 128$ pixel $\mathrm{T}_{2}{ }^{*}$ maps were calculated using a pixelwise linear fitting algorithm on the series of images. To compare elevation of mRNA, we constructed $\mathrm{R}_{2}{ }^{*}$ maps (the inverse of $\mathrm{T}_{2}{ }^{*}$ maps), which showed signal elevation with SPION retention.

Data analysis. We selected MR slices from MR data of each animal group (1.7-0.2 mm, relative to bregma) for $t$ test statistical analysis, but avoided those with partial volume artifacts in regions close to the olfactory bulbs and the nose. We used in-house software (Athinoula A. Martinos Center for Biomedical Imaging at MGH) to coregister the images of whole mouse brains, and then superimposed and averaged the $\mathrm{R}_{2}{ }^{*}$ maps within designated brain slices. A computer-generated scale is also included in the $\mathrm{R}_{2}{ }^{*}$ maps.

\section{Results}

\section{Characterization of SPION-sODN}

SPION has an average iron oxide core size of $\sim 10 \mathrm{~nm}$ and an average dextran coating thickness of $7 \mathrm{~nm}$, as measured by transmission electron microscopy and light scattering techniques. The design of SPION-sODN is presented in Figure $1 A$. Table 1 shows both longitudinal and transverse relaxivities ( $\mathrm{r} 1$ and $\mathrm{r} 2$, respectively) for SPION-NA, SPION-cfos (as one example for all SPIONsODNs), and unbound SPION; the SPION-NA and SPION-cfos relaxivities were lower than those of unbound SPION. The average volume-weighted diameter of SPION-cfos was $18 \mathrm{~nm}$ at $25^{\circ} \mathrm{C}$, and $20 \mathrm{~nm}$ at $37^{\circ} \mathrm{C}$. Addition of lipofectin to all SPION-sODN before infusion did not significantly change the core size, because $<1 \%$ measured larger in the presence of lipofectin (Table 1). We calculated the molecular weight of SPION-cfos to be $5.6 \times 10^{-19} \mathrm{~g}$ per nanoparticles. Each SPION-cfos contains $\sim 6000$ iron atoms, based on the relative viscosity of SPION-cfos (1.025 corresponding to 0.4 $\mathrm{mg} / \mathrm{ml}$ Fe concentration) in sodium citrate buffer at $\mathrm{pH} 8$ (Reynolds
$1 \mathrm{~A}$.
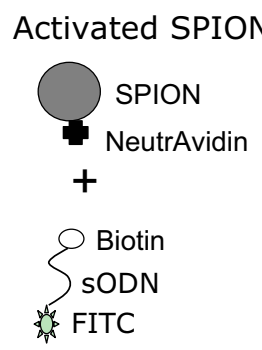

FITC-sODN-biotin

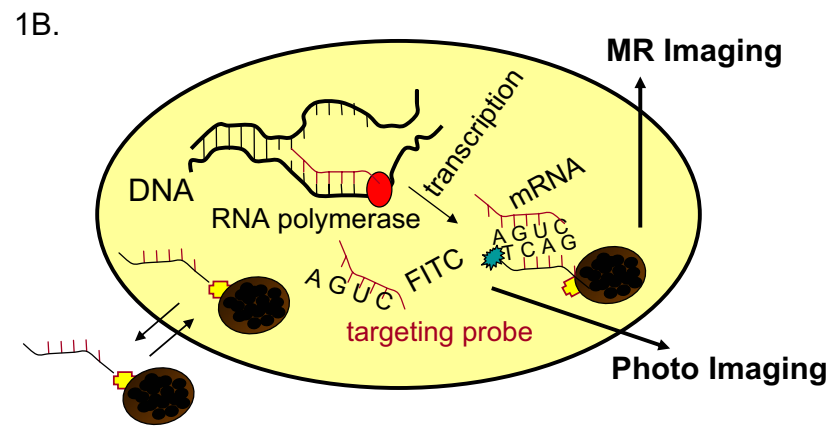

Non-targeting probe

Drawing not to scale

Figure 1. $\quad \boldsymbol{A}$ illustrates our probe design, and $\boldsymbol{B}$ illustrates a theoretical diagram for cellular mRNA targeting by SPION-SODN. FITC-sODN-biotin is conjugated to SPION-NA to generate SPION-SODN. The SODN can be labeled with an MR susceptibility agent, SPION, and/or with other imaging dye, such as FITC.

et al., 2005). The purity of SPION-NA is presented in the supplemental material (available at www.jneurosci.org). Two sets of evidence on FITC-sODN-biotin binding to SPION-NA are shown here: gel shift assay and Stern-Volmer plot.

\section{Gel mobility shift on binding}

We presented the electrophoretic migration of one example (SPION-cfos) in agarose gel. Under ordinary light source, SPION appears brown in color with intensity proportional to its concentration. Whereas three samples of sODN-bound SPION migrated toward the anode (Fig. 2A, lanes 1-3), unconjugated SPION-NA moved in the opposite direction or remained in the well (lane 4). Given that most of the conjugated SPION (SPIONsODN) samples in lanes 1-3 moved in the direction of the anode, as DNA should, it appears that DNA (charged molecules) pulls SPION-NA toward the anode. This finding illustrates strong complex formation between SPION-NA and FITC-sODN-biotin via avidin and biotin.

When illuminated with a light source that emits a wavelength at the secondary absorption maximum for FITC, the gel shows migration of free and unbound sODN at $11 \mathrm{~cm}$ in lane 1 , and the signal is so strong for $120 \mathrm{pmol}$ that it spills over to adjacent lanes (Fig. $2 B$ ). In lanes in which SPION-NA and FITC-sODN were conjugated and loaded (lanes 2 and 3), fluorescence signal appears at 1 and $11 \mathrm{~cm}$, with most of the dye traveling only $1 \mathrm{~cm}$, a distance similar to that traveled by SPION in Figure $2 \mathrm{~A}$, lanes $1-3$. This result indicates that there is a band shift typical of DNA-protein binding or conjugation of free sODN and SPION-NA via biotin on SODN and NA on SPION. The appearance of a smear between 1 and $11 \mathrm{~cm}$ reflects the size distribution of SPION-NA in Table 1. Variations in fluorescence exist between batches of SPION-NA in lane 2 (batch A, 17 months of age) 
and lane 3 (batch B, 2 months of age). The binding capacity remained with long shelf life, although it may show diminished signal intensity (supplemental Fig. S4, available at www.jneurosci.org as supplemental material).

To confirm the presence of SPION in the fluorescent dye of SPION-cfos samples, we measured the $T_{2}$ relaxation times in gel slices excised at $0,1,2$, and $3 \mathrm{~cm}$ from the well. Compared with the $\mathrm{T}_{2}$ relaxation time in the gel without samples (denoted as blank in Fig. 2C), the gel sample from unbound FITC-sODN-biotin (denoted as sODN in Fig. 2C) exhibited no change, indicating SPION was not present. However, Figure $2 C$ shows that the $\mathrm{T}_{2}$ relaxation times of gels containing SPION-NA were comparatively shortened in gel samples from above the well $(0 \mathrm{~cm})$ and $1 \mathrm{~cm}$ below the gel; $\mathrm{T}_{2}$ reduction in the $2 \mathrm{~cm}$ gel sample was slightly reduced. The shortened $\mathrm{T}_{2}$ relaxation times in the gel slice above the wells and at $1-3 \mathrm{~cm}$ in the gel containing SPION-sODN in Figure $2 C$ are consistent with fluorescence shift with SPION migration (that is, greater SPION and more T2 reduction) (Fig. $2 A, B$ ). The data demonstrate that SPION-sODN exhibits comigration as a single moiety. We showed binding at equal concentrations for SPIONNA, and FITC-sODN exhibited less fluorescence at $1 \mathrm{~cm}$ (Fig. 2 B) (also in supplemental Fig. S4, available at www.jneurosci.org as supplemental material), indicating a possible quenching effect when a transition metal is bound to a fluorescence moiety.

\section{Quenching efficiencies}

We observed reduction in FITC fluorescence emission when increasing SPION-NA was bound to FITC-sODN-biotin in repeated measurements (Fig. $3 A$ ). Using data in Figure $3 A$, we constructed a Stern-Volmer plot for SPION-sODN to examine the fluorescence quenching effect as a function of iron concentration. Figure $3 B$ demonstrates that the ratio of fluorescence inten$\operatorname{sity}\left(F_{0} / F q\right)$ to quencher concentration (iron) detected under various quencher concentrations is linear. The Stern-Volmer constant obtained for iron in SPION was $3.1 \times 10^{6} / \mathrm{M}$.

\section{Colocalization of SPION-NA and s-ODN for MR assessment}

To function as a reporter of gene transcription as we envisioned, (1) SPION-sODN must remain linked after delivery to the brain for uptake (Fig. $1 B$ ) and (2) SPION retention must be positively correlated with intracellular transcripts and revealed in MR assessment. To test this, we injected either unconjugated SPION or SPION-cfos into the left cerebral ventricle. If the probe complex remains intact, we expect colocalization of sODN and iron oxide in the same tissue areas. We acquired MRI in live animals at multiple time points after injection and then excised the brain tissue for histological examination of FITC-sODN-cfos and iron oxide $1 \mathrm{~d}$ after delivery. The presence of cerebral SPION and sODN were determined by two methods: (1) PB and NFR stains to detect intracellular iron and visualize the nucleus, respectively, and (2) fluorescent microscopy to detect FITC-labeled sODN.

\section{Turnover of SPION in mouse brains}

Figure 4 shows MR brain images from live animals obtained at magnetic field strength of 9.4 tesla. Compared with preinfusion brain images (Fig. $4 A$, baseline), MR signal was reduced from the infusion site across the midline to the right (contralateral) hemi-
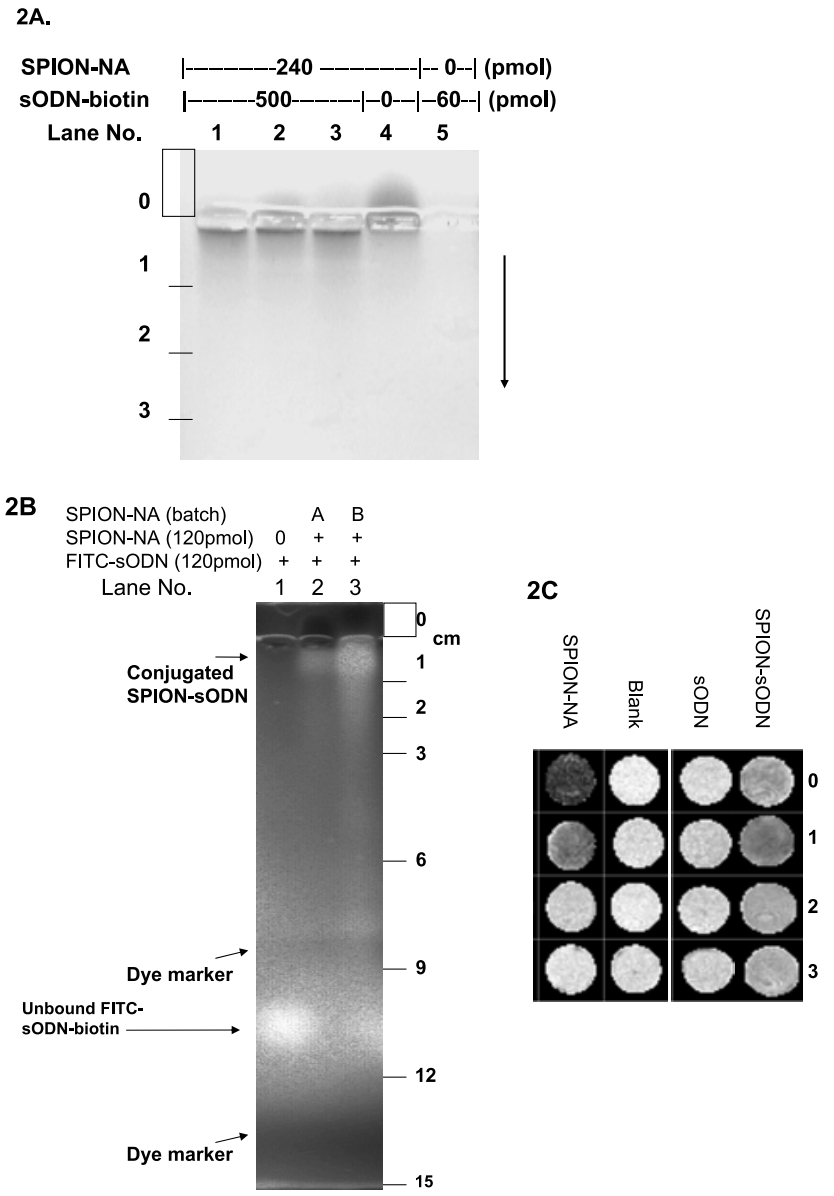

Figure 2. Linkage of SPION and SODN shows mobility shift during gel electrophoresis. $\boldsymbol{A}$ shows the migration of SPION-NA with (lanes 1-3) or without (lane 4) SODN, as acquired with an ordinary light source after gel electrophoresis at room temperature $(900 \mathrm{~V} \cdot \mathrm{h} ; 40 \mathrm{~V} / \mathrm{h})$. The arrow shows the direction of sODN migration. $\boldsymbol{B}$ shows FITC-sODN after electrophoresis ( 300 $\mathrm{V} \cdot \mathrm{h} ; 150 \mathrm{~V} / \mathrm{h}$ ) under a $302 / 365 \mathrm{~nm}$ light source (exposure time, $8 \mathrm{~s}$ ). The distance of elution from the well for each gel slice is shown in centimeters. SPION-NA of batch A is 17 months of age, and that of batch $B$ is freshly prepared ( 2 months). The quenching effect of SPION reduces the fluorescence of the FITC-sODN on SPION (saturation binding). The rows on the top of panels show the concentrations of components in each sample applied to the agarose gel. $\boldsymbol{C}$ shows $\mathrm{T}_{2}$ relaxation times in gel slices from $\boldsymbol{A}$ and $\boldsymbol{B}$.

sphere immediately ( $<30 \mathrm{~min}$ after SPION-cfos infusion at $1 \mu \mathrm{g}$ per mouse) (Fig. $4 B$, top row), distributed at least $4 \mathrm{~mm}$ in either direction from the infusion site, and covered the cerebellum and the olfactory ventricle (olfactory bulb), remaining detectable for 


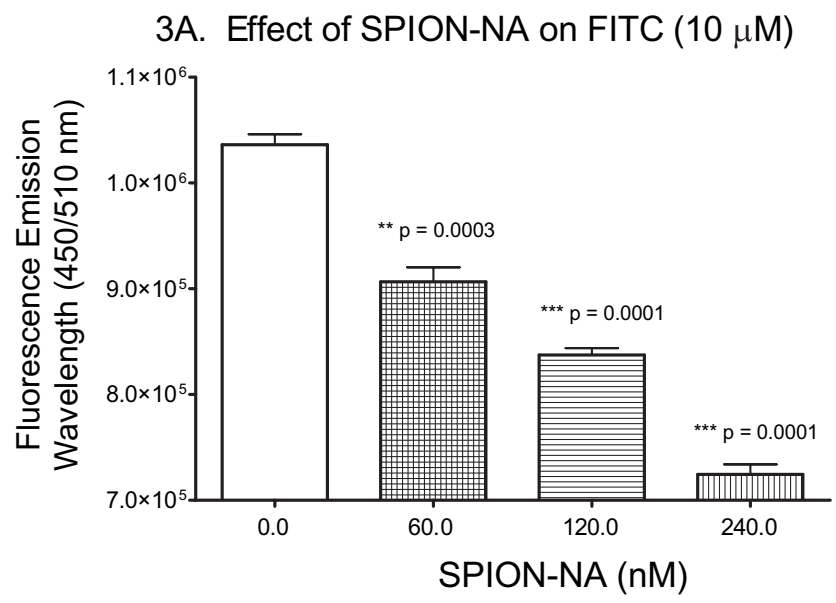

3B. Stern-Volmer plot

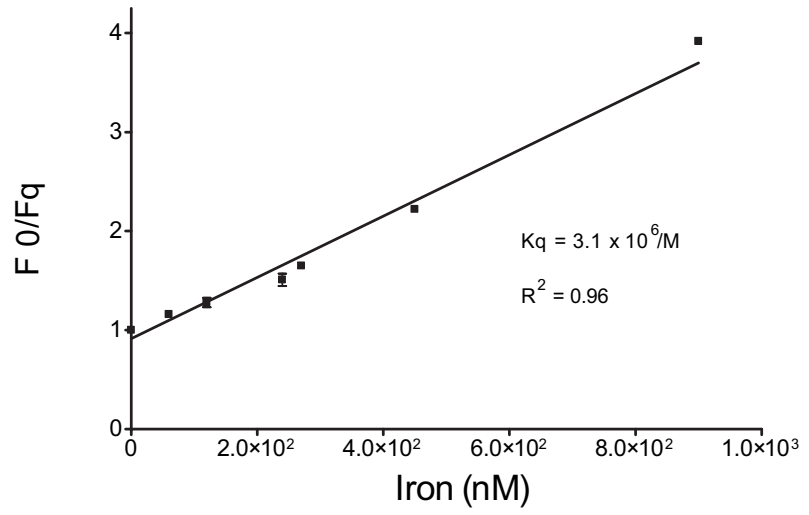

Figure 3. Quenching effect of SPION reduces fluorescence emission of the FITC-SODN on SPION. A shows decreasing fluorescence emission of FITC-SODN-biotin on binding to SPION-NA. Error bars indicate SEM. $\boldsymbol{B}$ shows a Stern-Volmer plot constructed for SPION-SODN. $K_{\mathrm{q}}$ is the Stern-Volmer constant calculated from the equation $F_{0} / F_{q}=1+K_{\mathrm{q}}[Q]$, where $F_{0}$ and $F_{\mathrm{q}}$ are fluorescence emission at zero $\left(F_{0}\right)$ and quencher concentration $\left(F_{\mathrm{q}}\right)$ of SPION-NA, and $Q$ is the concentration of SPION-NA (Murphy et al., 2004; Li et al., 2005).

at least $1 \mathrm{~d}$ after infusion (Fig. $4 \mathrm{~B}$, second row). However, relatively little $\mathrm{T}_{2}{ }^{*}$ signal reduction was observed in animals infused with unconjugated SPION at $1 \mu \mathrm{g}$ per mouse, except within the infusion track (Fig. 4C). When unconjugated SPION dose was increased to $5 \mu \mathrm{g}$; we observed T2 signal reduction at $30 \mathrm{~min}$ after infusion (Fig. 4CC, top rows). Unlike SPION-cfos, the majority of unconjugated SPION was no longer present, except in the lateral and fourth ventricles, $1 \mathrm{~d}$ after infusion (Fig. 4C, bottom row). SPION was not retained when infused in a mixture of SPION and FITC-sODN-biotin (Fig. 4D), indicating that linkage is important for SPION retention. While unconjugated SPION is rapidly cleared in the cerebral ventricles, SPION-cfos provides an extended window for MR imaging.

\section{Histology: SPION-cfos uptake}

Gross examination of PB and NFR stains revealed that Prussian blue staining was stronger in the ipsilateral hippocampus than in the contralateral hippocampus in animals that received SPIONsODN-FITC (Fig. 5, compare $A, B$ ). There is weak yet positive signal showing colocalization of sODN and iron in the ipsilateral hippocampus in adjacent postmortem samples (Fig. $5 A, C$, respectively). At high magnification (Fig. $5 A, B$, inset panels), we noted particles $1 \mu \mathrm{m}$ or less in size in the cytoplasm and in regions surrounding the nucleus; cytosolic PB stain was observed as small aggregates (Fig. 5A, B, inset panels, arrows). Figure 5D demonstrates a lack of iron oxide staining in animals that received unconjugated SPION; these animals exhibited no MR signal reduction (Fig. 4C).

Histology: colocalization of iron oxide and SPION-cfos

Figure $5 E$ shows sODN uptake in neurons and their axons (long arrow) between the ventricle and CA neurons (indicated by brackets). We also observed FITC-sODN uptake by cells in the vascular wall (Fig. 5E) and in the CA neuronal formation (Fig. $5 G$, brackets). Figure $5 F$ shows background fluorescence in the control sample (baseline) of animals that received no infusion. We demonstrate that cells that take up SPION-cfos-FITC are not glia, but neurons (Fig. 5G).

Given the quenching effect of SPION on FITC-sODN, we modified the method by using FITC-IgG against digoxigenin (dig) to detect the presence of sODN with sODN-dig. We infused SPION-cfos-dig ( $2 \mu \mathrm{g}$ per mouse) with and without lipofectin and allowed $1 \mathrm{~d}$ for uptake. Figure $5, H$ and $I$, shows that SODN is present in the cortex and in the CA neuronal formation (green photomicrograph images were converted to black and white with Photoshop software) regardless of whether the infusion included lipofectin. Tissue adjacent to that shown in the inset of Figure $5 I$ (Fig. 5J) also demonstrates the presence of iron oxide in the ventricular wall (asterisk), CA neuronal formation (CA1), dentate gyrus (DG), and vascular wall in the hippocampus. Figure $5 K$ shows PB staining in the cerebellum, including in the Purkinje neurons. The presence of cortical iron oxide has been reported (Liu et al., 2007). The data show that iron oxide and sODN travel at least $1.5 \mathrm{~mm}$ (at the hippocampus) to $6 \mathrm{~mm}$ (at the cerebellum) from the infusion site.

\section{MR assessment of SPION-cfos correlates with histology of} iron oxide

To compare MR assessment to histology with PB stain in Figure $5, A$ and $B$, we measured regional $\mathrm{T}_{2}{ }^{*}$ relaxation time (in milliseconds) at regions of interest (ROIs) in the hippocampus. Compared with the preinfusion baseline hippocampus $\left(\mathrm{T}_{2}{ }^{\star}\right.$-weighted image shown in Fig. $4 A a)$, there was no significant difference between regional $\mathrm{T}_{2}{ }^{*}$ relaxation times $(40 \mathrm{~ms}$ ) of the left and right hippocampus of animals that received unconjugated SPION. In the same ROI of the left (ipsilateral) (LH) and right (contralateral) $(\mathrm{RH})$ hippocampus of animals that received SPION-cfos, regional $\mathrm{T}_{2}{ }^{*}$ relaxation times were reduced to 7 and $17 \mathrm{~ms}$, respectively (image shown in Fig. $4 \mathrm{Bb}$ ), suggesting that SPION-cfos is retained in the left hemisphere. Therefore, MR images in Figure $4 \mathrm{~B}$ correctly reflect regional iron content corresponding to histology of two hemispheres of the same animal brain shown in Figure 5, $A$ and $B$. The same was observed in three additional brains (data not shown).

\section{SPION-sODN report endogenous gene transcript in}

\section{live animals}

The hippocampus showed the highest uptake within any given brain (Fig. $4 B$ ), but we avoided analyzing the hippocampus among different animals for the following reasons. First, given the very small size of the hippocampus and its location in the ventricle, it is very easy to include regions that exhibit ventricular SPION yet to be taken up. Second, the hippocampus contains the hippocampal fissure, where SPION may be detected before its uptake/distribution. We selected the contralateral somatosensory cortices (SSCs) as our ROI for analysis because it is distant from the ventricle and the air-surface interface of the trachea 
(ROI outlines overlay on MR anatomical images). We converted $\mathrm{T}_{2}^{*}$ relaxation times to $\mathrm{R}_{2}^{*}\left(\mathrm{R}_{2}^{*}=1 / \mathrm{T}_{2}{ }^{*}\right) ; \mathrm{R}_{2}{ }^{*}$ values were used here for statistical analysis of SPION retention. Figure $6 A$ shows statistical analysis of SPION retention in the contralateral SSC of animals that received conjugated and unconjugated SPION probes. Animals that received unconjugated SPION did not exhibit significantly elevated $\mathrm{R}_{2}{ }^{*}$ values at all time points compared with baseline. SPION retention in animals that received SPION- $\beta$ actin and SPION-cfos was significantly higher than baseline as early as $7 \mathrm{~h}$ after injection. $\mathrm{R}_{2}{ }^{*}$ values peaked at $38.5 \mathrm{~s}^{-1} 1 \mathrm{~d}$ after infusion in the contralateral SSC of the animals that received SPION-cfos (Fig. 6A), and returned to baseline values within $2 \mathrm{~d}$ (data not shown). We therefore concluded that the clearance time (the time frame spanning the point of infusion to the point at which $\mathrm{R}_{2}{ }^{*}$ values returned to baseline) was $2 \mathrm{~d}$ for the $1 \mu \mathrm{g}$ dose of SPION-cfos. The $\mathrm{R}_{2}{ }^{*}$ values in the contralateral SSC of animals that received SPION- $\beta$ actin $(1 \mu \mathrm{g})$ peaked at, and were significantly higher than those of the SPION-cfos group $7 \mathrm{~h}$ after infusion, and remained significantly elevated for at least $1 \mathrm{~d}$.

We compared SPION retention to gene transcript maps using in situ hybridization in normal mouse brain samples. The intensity of c-fos mRNA was barely detectable (Fig. 6B) and the mRNA maps of $\beta$-actin mRNA were higher than those of c-fos mRNA in normal mouse brains. The intensity of $c$-fos and $\beta$-actin mRNA, shown in Figure 6, $B$ and $C$, respectively, agrees with SPION retentions of SPION$\beta$ actin and SPION-cfos in the contralateral SSC, $7 \mathrm{~h}$ after infusion, as shown in Figure $6 \mathrm{~A}$. The data suggest that SPION retention in normal live mice is positively correlated to intracellular mRNA targets of normal mouse brain as hypothesized in Figure $1 B$.

\section{Elevation of SPION retention after acute amphetamine infusion}

We wanted to demonstrate that SPION retention would increase with elevated gene transcripts in those animals treated with targeting SPION-sODN, but not in those infused with nontargeting SPION-Ran (a SPION with sODN of random sequence and therefore no cellular target). We first compared the retention profiles for SPION-cfos and SPION-Ran, both in the same dose, in the striatum of the forebrain (ROI outlines overlay on MR anatomical images), where amphetamine had previously been shown to stimulate expression of c-fos mRNA (Schmauss, 2000; Conversi et al., 2004). SPION retention, measured as $\mathrm{R} 2^{\star}$ values, for SPION-Ran in normal brain was within the baseline values (Fig. 6D). SPION-cfos retention was slightly but significantly elevated relative to SPION-Ran $7 \mathrm{~h}$ after infusion $(p=0.004)$. These results suggest that the differences in SPION retention 4a. Baseline No Infusion

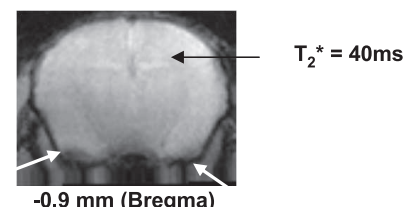

$-0.9 \mathrm{~mm}$ (Bregma)

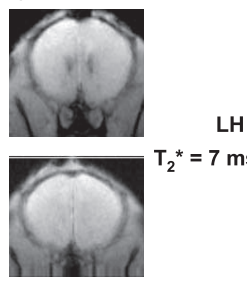

4b. SPION-cfos, one day

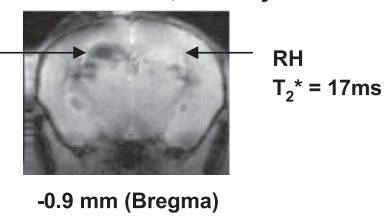

infusion slice $\quad 2 \mathrm{~mm}$

$-0.9 \mathrm{~mm}$ (Bregma)

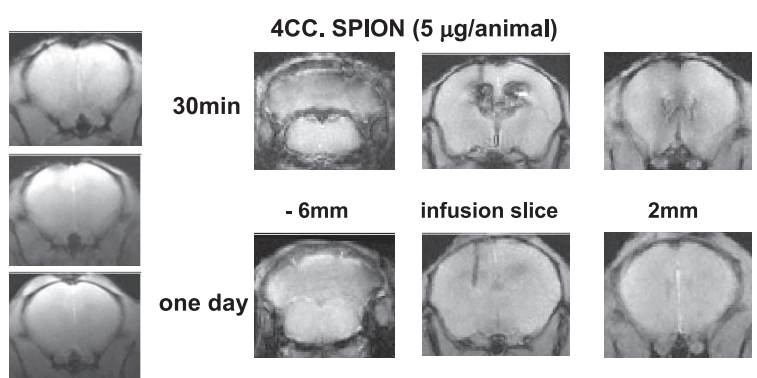

4D. SPION and sODN-cfos mixture, no linkage ( $5 \mu \mathrm{g} / \mathrm{animal})$

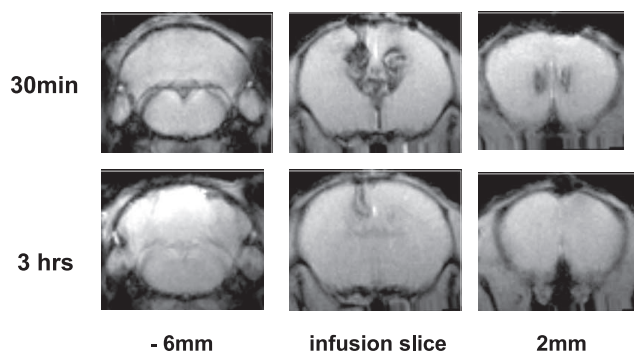

$2 \mathrm{~mm}$

Figure 4. SPION-cfos probe provides an extended window for MRI in live mice. Images acquired at TR/TE $=500 / 6 \mathrm{~ms}$ are shown $(N=4$ each). Images were acquired from one representative animal from each probe group. Images include brain regions ippocampus. $\boldsymbol{C}$ and $\boldsymbol{C}$ show MR images of two representative animals after unconjugated SPION; insets $\boldsymbol{a}$ and $\boldsymbol{b}$ show MR brain slices from the posterior to the infusion site at $-0.9 \mathrm{~mm}$ (bregma) in the same animal as shown in $\boldsymbol{A}$ and $\boldsymbol{B}$, respectively. $\boldsymbol{D}$ shows MR images after infusion of unconjugated SPION, or mixture of SPION and SODN without NA and biotin linkage. between targeting and nontargeting probes were distinguishable using MRI in the contralateral striatum $7 \mathrm{~h}$ after intracerebroventricular infusion.

The induction of immediate-early genes by amphetamine is shown using this novel probe and method of detection with MRI. Figure 7 shows $\mathrm{R}_{2}{ }^{*}$ maps of C57Black6 mice treated with either amphetamine or saline. No elevation in $\mathrm{R}_{2}{ }^{*}$ was seen in animals that received SPION-Ran, whether with or without acute amphetamine administration (Fig. 7A). Animals that received SPION-cfos and amphetamine exhibited robust elevation of $\mathrm{R}_{2}{ }^{*}$ in the forebrain (Fig. 7B), particularly in the nucleus accumbens (NAc); $\mathrm{R}_{2}{ }^{*}$ elevation was less robust in the striatum $(\mathrm{CPu})$ and cingulate and prelimbic/infralimbic cortices [collectively the medial prefrontal cortex (mPFC)], and only little elevation was seen in the SSCs. In the same animals, we observed a gradient of in- 


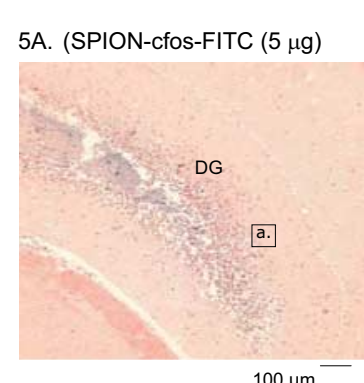

5C. SPION-cfos-FITC (ipsilateral)

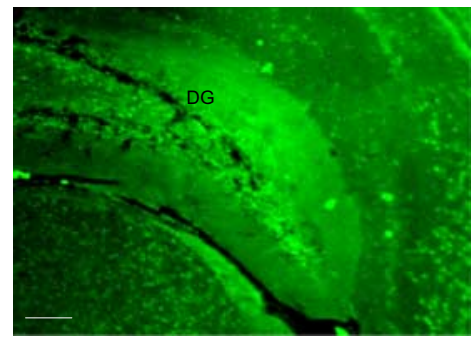

$170 \mu \mathrm{m}$

5H. Lipofectin

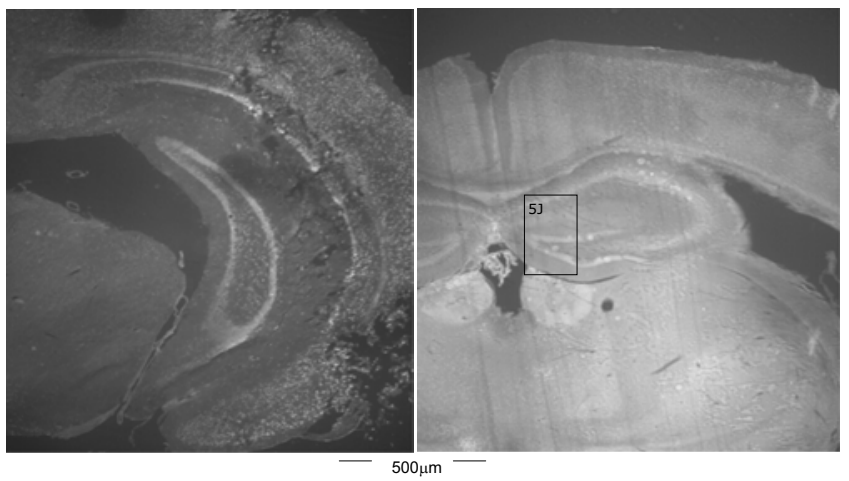

5B. Contralateral

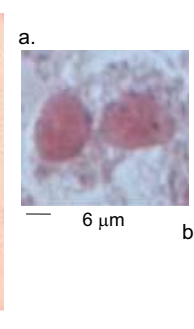

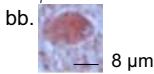

bb.

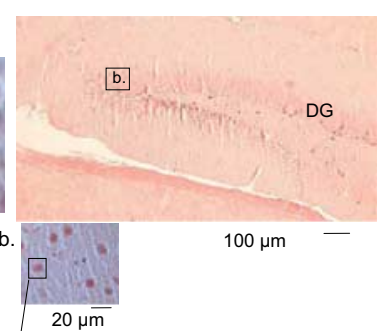

5D. Unconjugated SPION $(5 \mu \mathrm{g})$

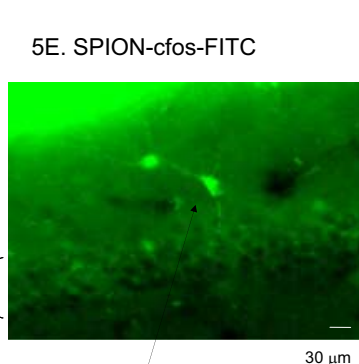

5F. Baseline, no infusion

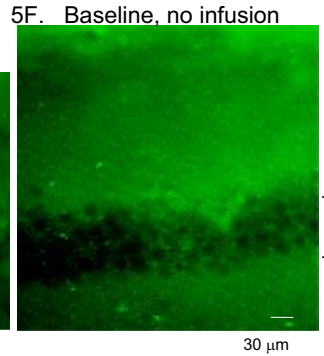

5G.SPION-cfos-FITC and GFAP stain

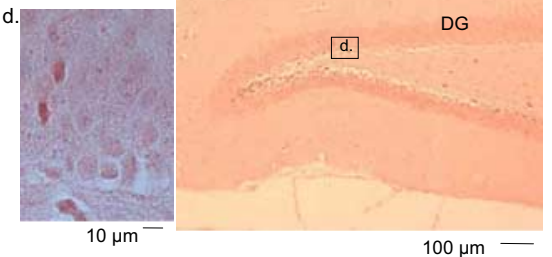

FITC in

neurons and their axons

glia

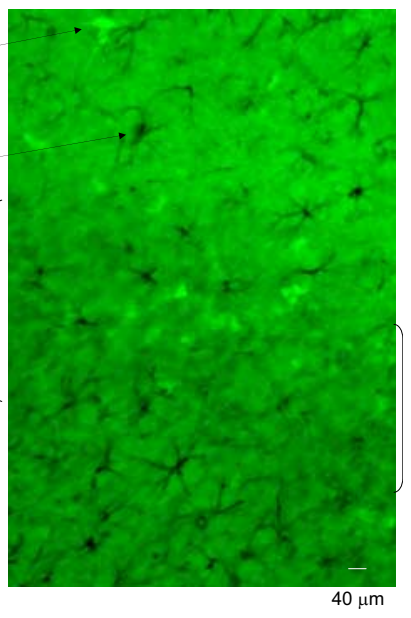

$5 \mathrm{~K}$. Transverse section of a cerebellar folium Molecular layer Purkinjie layer
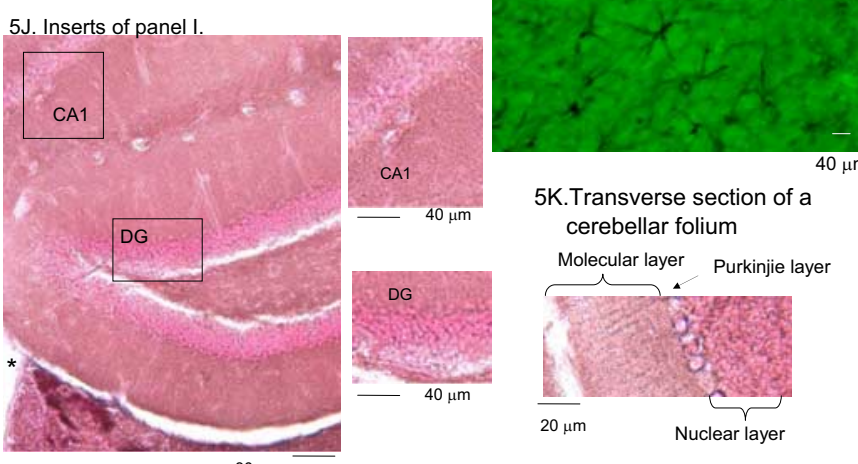

$20 \mu \mathrm{m}$

Nuclear layer

$80 \mu \mathrm{m}$

Figure 5. Colocalization of SODN-cfos and iron oxide in mouse brains $1 \mathrm{~d}$ after infusion of SPION-cfos. Animals were given an infusion of SPION-cfos-FITC ( $A-C, 1 \mu \mathrm{g}$ of Fe per mouse) or unconjugated SPION ( $\boldsymbol{D} ; 5 \mu \mathrm{g}$ of Fe per mouse), all with lipofectin $(N=4$ in each group). Brain tissue was obtained $1 \mathrm{~d}$ later. PB stain and NFR counterstain are shown in $\boldsymbol{A}, \boldsymbol{B}$, and $\boldsymbol{D}(10 \times$ objective) and in the insets $\boldsymbol{a}, \boldsymbol{b}, \boldsymbol{b} \boldsymbol{b}$, and $\boldsymbol{d}(100 \times)$. $\boldsymbol{A}$ and $\boldsymbol{C}$ show adjacent tissue slices. $\boldsymbol{C}$ and $\boldsymbol{E}$-I show fluorescent micrographs. $\boldsymbol{E}$ shows FITC-sODN-cfos in brain samples from animals that were given infusion of SPION-cfos-FITC ( $1 \mathrm{~d}$ after infusion); $\boldsymbol{F}$ shows the baseline control. Tissue in $\boldsymbol{G}$ was from the same group of animals as in $\boldsymbol{C}$ and $\boldsymbol{E}$ but was treated with antibodies against glial fibrillary acidic protein (GFAP) and DAB stains. Hand Ishow sODN uptake in the contralateral hemisphere of the animals infused with SPION-cfos-dig ( $2 \mu \mathrm{g}$ of Fe per mouse) with $(\boldsymbol{H})$ and without $(\boldsymbol{I})$ lipofectin $(N=3$ each). Presence of sODN-dig $1 \mathrm{~d}$ after infusion was detected using FITC-IgG against dig (Cui et al., 1999). $\boldsymbol{J}$ and $\boldsymbol{K}$ show SPION uptake in the hippocampus and the cerebellum of the same animal as in $\boldsymbol{I}$. Purkinje neurons are located in the Purkinje layer as aligned, stacked cells with a large nucleus $(\boldsymbol{K})$. The asterisks show stain on the surface of ventricular wall $(\boldsymbol{J})$. All images except those in $\boldsymbol{E}$ and $\boldsymbol{F}$ were acquired from tissue sections treated with xylene. $\boldsymbol{E}$ and $\boldsymbol{F}$ show non-dehydrated tissue. Tissue thickness was $20 \mu \mathrm{m}(\boldsymbol{A}-\boldsymbol{D}, \boldsymbol{H}, \boldsymbol{I})$ or $100 \mu \mathrm{m}(\boldsymbol{E}-\boldsymbol{G}, \boldsymbol{J}, \boldsymbol{K})$, which allows us to observe axons (long arrows; $\boldsymbol{E}$ ) and vascular wall $(\boldsymbol{E}, \boldsymbol{J})$. The brackets in $\boldsymbol{E}-\boldsymbol{G}$ point to the $(A 1$ neuronal formation in the hippocampus. DG, Dentate gyrus.

creasing $\mathrm{R}_{2}^{*}$ values from the dorsolateral toward ventromedial striata. These results are consistent with distinct patterns of Fos expression in the forebrains of C57Black6 mice associated with acute amphetamine stimulation (Conversi et al., 2004).

Figure $7 C$ demonstrates no significant induction of c-fos expression in the SSC, but a significant elevation in all three ROIs in animals that received SPION-cfos and amphetamine. Because $\mathrm{R} 2{ }^{\star}$ values in the SSC were not significantly elevated in all treatments, we concluded that our measurements were not significantly influenced by artifacts located outside the brain. Other support comes from control animals that received SPION-Ran, for which we observed no significant difference in the $R_{2}$ of all ROIs (data not shown). Our observations are consistent with the previously reported increases in c-fos mRNA after amphetamine.

\section{Discussion}

Assays of gene transcription often use nucleic acid probes complementary to their mRNA targets in autopsy specimens. Recent developments in contrast-enhanced and ligand-targeted MRI techniques have made these novel tools for assessing the delivery of biological agents and detecting biological markers in living subjects. DNA-based probe may offer greater variety in aptamer design (Liu et al., 1994; Perez et al., 2002). Among imaging modalities, MRI offers less-invasive image acquisition and enhanced image resolution, and has the potential to become an important tool for assessing delivery of exogenous agents and detecting biological markers in living subjects (Roberts et al., 2000).

We have synthesized several SPION-linked DNA aptamers and demonstrated their application for detecting mRNA in the brains of living animals. Our study focused on one aptamer having sequence complementary to the well characterized c-fos mRNA of neuronal origin (Sagar et al., 1988). The delivery, uptake, and hybridization to mRNA of this ODN in rodent brains have been reported (Liu et al., 1994; Cui et al., 1999; Y. Zhang et al., 1999). When administered at a dose of $1 \mu \mathrm{g}$ of Fe per mouse via intracerebroventricular injection, SPION-cfos is diffused throughout the brain and selectively retained, as confirmed by 
A

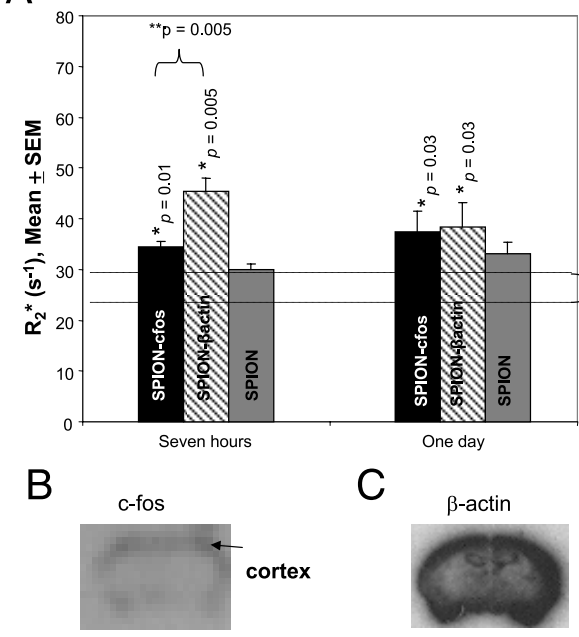

D

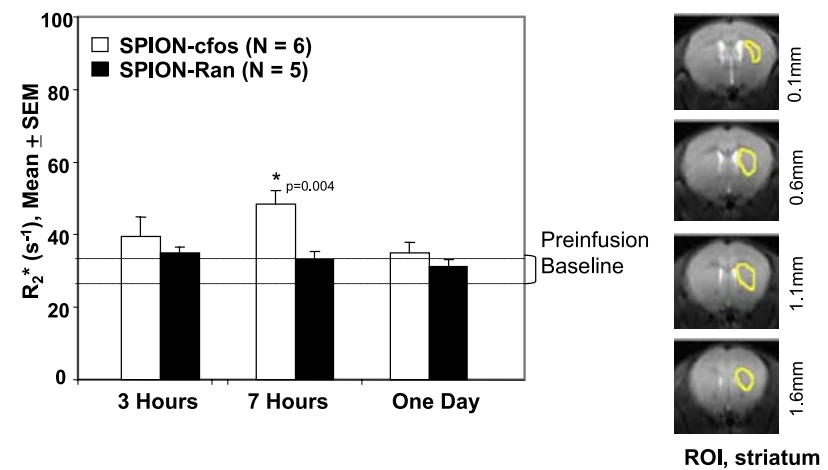

Figure 6. Temporal profiles of SPION retention in $\mathrm{R}_{2}{ }^{*}$ maps. $A$ shows animals infused with SPION-cfos, SPION- $\beta$ actin, or unconjugated SPION ( $1 \mu \mathrm{g}$ of Fe per mouse; $N \geq 4$ each). Multiple cerebral MRIs were acquired as GE MRI in live animals at indicated time points. Preinfusion data serve as baseline (see examples in Fig. 5 for images or saline groups in Fig. 7 for $R_{2}{ }^{*}$ maps). We calculated mean $R_{2}{ }^{*}$ values in an $R O I$ in contralateral SSC to determine SPION retention. No significant differences in $\mathrm{R}_{2}{ }^{*}$ values were observed in baseline and animals that received SPION. The $p$ values denote mean $\mathrm{R}_{2}{ }^{*}$ values that are significantly different from baseline $\left({ }^{*}\right)$ or significant differences between SPION-cfos and SPION- $\beta$ actin ${ }^{* *}$ ), calculated using a $t$ test. Based on the mean and SEM from $7 \mathrm{~h}$ after infusion, and because power calculation reveals that four animals per group are needed to obtain a statistically significant difference for the effect sizes seen here with $95 \%$ confidence and $p=0.01$, we measured five mice in each group. The ROls are shown at right. $\boldsymbol{B}$ and $\boldsymbol{C}$ show mRNA maps of $\mathrm{c}-\mathrm{fos}(\boldsymbol{B})$ and $\beta$-actin $(\boldsymbol{C})$ gene transcripts in a normal mouse (no infusion) using in situ hybridization and a ${ }^{32} \mathrm{P}$-labeled antisense RNA probe (Cui et al., 1999a) $(N=4)$. D shows the profile of SPION retention in the contralateral striatum. Regional SPION retention of SPION-cfos and SPION-Ran (1 $\mu \mathrm{g}$ of Fe per mouse) is shown in the contralateral striatum for ROIs depicted to the right. Error bars indicate SEM.

histological assays of iron oxide and immunohistochemical detection of ODN, and corroborated by MRI. SPION unmodified or in mixture with (not linked to) sODN is not retained in the brain, even when given at a dose as high as $5 \mu \mathrm{g}$ of Fe per mouse. However, sODN (cfos) has been shown to initially attach to the ventricular wall, before appearing in the parenchyma, where it binds to c-fos mRNA (Liu et al., 1994; Cui et al., 1999). The exact routes of uptake and distribution from the ventricle are not well understood, but to enter the brain parenchyma, the probe must migrate across a number of well defined barriers such as the Virchow-Robin space, the perivascular lymphatic space between the vessels of the CNS, and the glial membrane on the brain surface between the parenchyma and ventricle wall. We demonstrate possible axonal transport of SPION-sODN (Fig. 5E), an observation that is consistent with reports using other MR con-
7A. $\quad \mathbf{R}_{\mathbf{2}}$ * Maps, SPION-Ran $(0.7 \mu \mathrm{g} /$ animal)

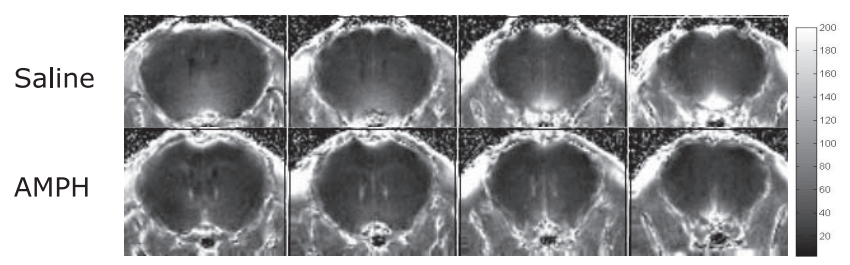

7B. $\quad \mathbf{R}_{\mathbf{2}} *$ Maps, SPION-cfos $(0.7 \mu \mathrm{g} /$ animal $)$
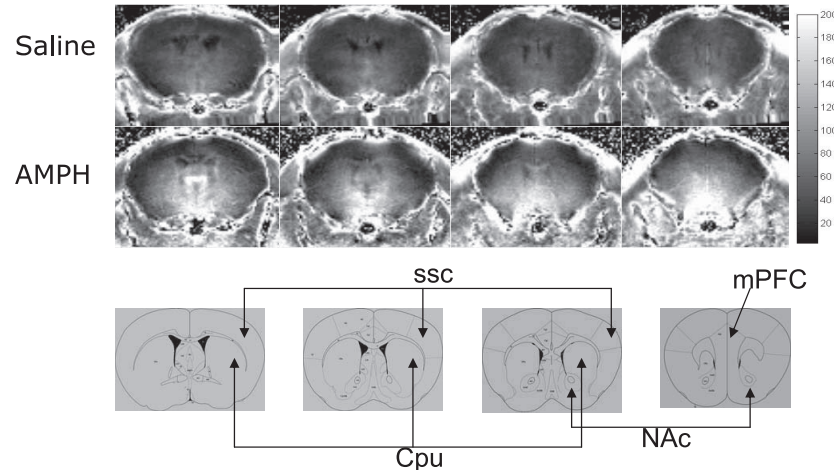

7C. cfos expression by SPION-cfos and MRI

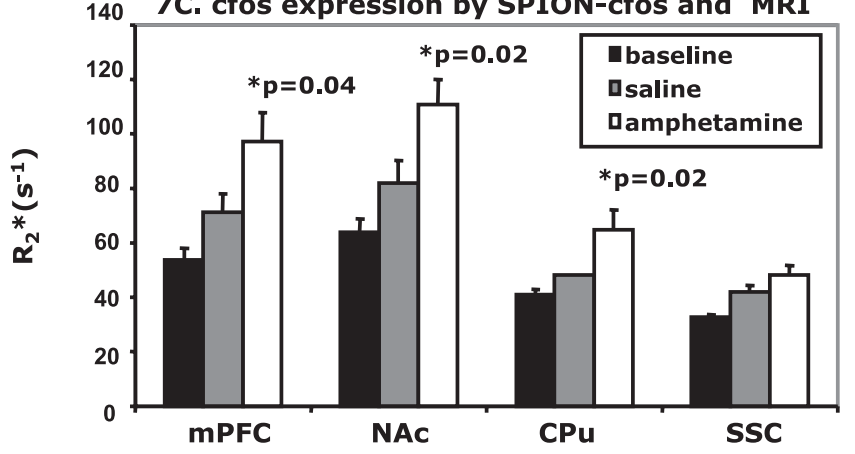

Figure 7. Specific elevation of $c$-fos $m R N A$ in $R_{2}{ }^{*}$ maps after acute amphetamine exposure. SPION-Ran $(N=5$ pairs; $A)$ or SPION-cfos $(N=6$ pairs; $\boldsymbol{B})$ was infused as in Figure 5 , except that amphetamine ( $4 \mathrm{mg} / \mathrm{kg}$ body weight) or saline $(10 \mathrm{ml} / \mathrm{kg}$ ) was administered intraperitoneally $4 \mathrm{~h}$ later, and MRI was acquired $3 \mathrm{~h}$ thereafter. Representative $\mathrm{R}_{2}{ }^{*}$ maps $\left(0-200 \mathrm{~s}^{-1} \mathrm{R}_{2}{ }^{*}\right.$ values are shown in the scale bar) are from four MR slices (same as Fig. $6 D$ ) in one randomly selected animal from each group. Schematic diagrams of anatomic features are within $0.25 \mathrm{~mm}$ of each MR slice (Paxinos and Franklin, 2001). Cshows profiles of SPION retention in ROIs from $\mathrm{R}_{2}{ }^{*}$ maps (see Fig. 6).mPFC, Medial prefrontal cortex; NAc, nucleus accumbens; CPu, caudate/putamen.

trast agents (Enochs et al., 1993; Filler, 1994; van Everdingen et al., 1994). In the current study, the uptake and distribution of ODN and iron in regions distant from the site of infusion are similar with lipofectin (Fig. 5A-J) and without lipofectin (Fig. $5 I-K)$. Using MRI, we showed that SPION-cfos permeated at least $8 \mathrm{~mm}$ in the ventricular area at the initial scan (Fig. 4), and the probe complex remained intact in samples taken $1 \mathrm{~d}$ after infusion (Figs. 5, 6). It can appear as the blooming of concentrated $\mathrm{T}_{2}$ agent. However, we submit that this "blooming effect" will be a very special one because it occurs only in SPION-sODN, not in the unconjugated SPION (with lipofectin) at the dose that we detect gene expression.

The fate of intracellular SPION-sODN depends on the sequence of targeting probe and the physical linkage between the two components, because neither the control probe without a target (SPION-Ran), or unconjugated SPION shows significant retention (Figs. 4-7A). Therefore, the presence of SPION-cfos and SPION- $\beta$ actin in the contralateral hemisphere indicates that retention is specific for targeting SPION-sODN beyond the infusion site. We show evidence that SODN binds to intracellular 
mRNA (Liu et al., 1994) and serves as a primer for specific cDNA reverse transcription (our unpublished observations). Binding of targeting SPION-sODN to its mRNA may create SPION retention (or exclusion delay) by a target-dependent mechanism (Fig. 6). Therefore, the larger aggregates seen under light microcopy with $\mathrm{PB}$ staining can be a compartmentalization of iron oxide awaiting exclusion. Compartmentalization could involve endosomes or other cytoplasmic structure (Arbab et al., 2005). This mechanism would explain the elevation of SPION at $7 \mathrm{~h}$ for targeting probe (SPION-actin and SPION-cfos) but not nontargeting probe (SPION-Ran).

It has been reported that SPION particles (Combidex; 833 $\mathrm{pmol} / \mathrm{kg}$ ) do not remain in rat brains beyond $3 \mathrm{~h}$ (Muldoon et al., 2004), although oligodendroglial cells have been reported to take up an iron oxide-derived MR contrast agent (Bulte et al., 1999). Magnetic nanoparticles covalently attached to DNA were first described by Perez et al. (2002) for detecting DNA-DNA interactions in cellular extracts. We show here a more facile, modular, and universal approach for generating MR reporters for different mRNA targets with DNA-RNA interaction. SPION-cfos and SPION- $\beta$ actin, at concentrations approximately one-tenth less than that used by Muldoon et al. (2004), are found to be stable and provide an extended window for MR imaging for at least 7-24 h after infusion. Although all types of brain cells are able to take up sODN (Ogawa et al., 1995), we have shown that retention of SPION-cfos and SPION- $\beta$ actin could be mediated by intracellular mRNA (Fig. 6). These characteristics will be investigated in the future with different probes for different cellular targets.

The intracerebroventricular technique we used to deliver this probe is a convection-enhanced delivery method (Sandberg et al., 2002), and the distribution pattern of SPION-linked ODN delivered by the technique is different from those observed with the use of a viral vector (de Marco et al., 1998; Hommel et al., 2003), in that cellular uptake and hybridization to target mRNA is required for our probe to function as a reporter. The intracranial method of probe delivery results in more uniform distribution with greater reproducibility of uptake and wider reaches into regions away from the infusion site. The presence of SPION in the contralateral hemisphere in animals that received SPION-cfos suggests that distribution of SODN-conjugated SPION is not limited to the infusion site. This delivery method is an improvement over other intracerebral injection techniques (Chiasson et al., 1992; Wahlestedt et al., 1993; Morrow et al., 1999; M. Zhang et al., 1999; Tolliver et al., 2000), as evidenced by the fact that the probes are distributed at least $8 \mathrm{~mm}$ anterior-posteriorly (Fig. $4 \mathrm{~B}$ ), and that SPION-cfos migrates to the contralateral hemisphere (Figs. $4 B, 5 B)$, possibly by way of the ventricular cerebrospinal fluid, to the perivascular space before axonal transport (Fig. $5 E$ ). Therefore, this probe is definitively an improvement over neuronal transport in the guinea pig facial nerve (Petropoulos et al., 1995).

Our delivery method, although invasive for clinical purposes, is appropriate for preclinical neurological assays in drug discovery. Uptake using intracerebroventricular delivery is robust; it is most suitable for applications in preclinical testing, and for use in antisense strategies for gene knock-down, gene therapies, and stem cell tracking. Other methods such as intravenous or intraperitoneal delivery coupled with opening of the blood-brain barrier $(\mathrm{BBB})$ induced by osmotic shock, severe stroke, or trauma, and attaching probes to antibodies against the surface protein of the BBB can presumably be used. However, successful delivery of probes by these methods, although less invasive, is limited to the regions in which the $\mathrm{BBB}$ is disrupted by osmotic shock (mannitol), or is often disease dependent (stroke and trauma). The intracerebroventricular technique is far more efficient and cost ef- fective, and can be compared with lumbar puncture in larger animals when proof of concept for the probe is successfully demonstrated by intracerebroventricular delivery and MR imaging in live animals.

Most importantly, we have observed colocalization of sODN and iron oxide in tissue samples taken $1 \mathrm{~d}$ after intracerebroventricular infusion; this observation is corroborated by MR signal assessments. We have not been able to show colocalization of sODN-cfos-FITC in the contralateral hemisphere with iron oxide, possibly because of the quenching effect of SPION on FITC, and we are developing a new detection method to overcome this limitation (Fig. 5H,I). However, iron oxide and sODN-cfos (or sODN- actin) could be located in the same cells; retention of SPION-cfos and SPION- $\beta$ actin is mRNA dependent (Fig. 6A,B). Because the half-life of c-fos mRNA, as determined in cell culture, is only $\sim 10$ min (Kabnick and Housman, 1988), the enhanced stability of SPION retention at $7-24 \mathrm{~h}$ in the brains of animals that re-

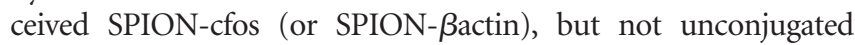
SPION or SPION-Ran, suggests the extended window of MR imaging may be a result of heteroduplex formation between mRNA and targeting SPION-sODN. This is supported by the fact that sODNcfos binds to c-fos mRNA both in vitro and in vivo, especially in the neuronal formation where FOS antigens are present in abundance (Liu et al., 1994). Furthermore, SPION-cfos forms a hybrid with c-fos mRNA in mouse brains (our unpublished observations). Lastly, elevated SPION retention in the forebrains of animals that received SPION-cfos, but not those that received SPION-Ran after acute administration of amphetamine corroborates elevation of c-fos mRNA.

Amphetamine alters the expression of several genes. The exact action of amphetamine is not totally understood, but it stimulates brain cells through several membrane receptors in the striatum and induces signal transduction by phosphorylation of cAMP response element binding protein (CREB). CREB activates the transcription of several genes, one of which is c-fos (Konradi et al., 1994; Hyman and Malenka, 2001). We also detected mRNA elevations in MRI using SPION-sODN probes in additional disease models (our unpublished observations). In the current study, the elevation of c-fos mRNA by amphetamine increases the hybridization with SPION-cfos probe and $\mathrm{R}_{2}{ }^{*}$ values in regions that show neuronal activation (Fig. 7). In conclusion, we designed target-specific RNA sensors and demonstrated their usefulness for in vivo monitoring of gene transcripts by time-lapse imaging of live animals.

\section{References}

Arbab AS, Wilson LB, Ashari P, Jordan EK, Lewis BK, Frank JA (2005) A model of lysosomal metabolism of dextran coated superparamagnetic iron oxide (SPIO) nanoparticles: implications for cellular magnetic resonance imaging. NMR Biomed 18:383-389.

Bulte JW, Zhang S, van Gelderen P, Herynek V, Jordan EK, Duncan ID, Frank JA (1999) Neurotransplantation of magnetically labeled oligodendrocyte progenitors: magnetic resonance tracking of cell migration and myelination. Proc Natl Acad Sci USA 96:15256-15261.

Bulte JW, Duncan ID, Frank JA (2002) In vivo magnetic resonance tracking of magnetically labeled cells after transplantation. J Cereb Blood Flow Metab 22:899-907.

Chiasson BJ, Hooper ML, Murphy PR, Robertson HA (1992) Antisense oligonucleotide eliminates in vivo expression of c-fos in mammalian brain. Eur J Pharmacol 227:451-453.

Conversi D, Orsini C, Cabib S (2004) Distinct patterns of Fos expression induced by systemic amphetamine in the striatal complex of C57BL/ 6JICo and DBA/2JICo inbred strains of mice. Brain Res 1025:59-66.

Cui J, Holmes EH, Liu PK (1999a) Oxidative damage to the c-fos gene and reduction of its transcription after focal cerebral ischemia. J Neurochem 73:1164-1174.

Cui JK, Hsu CY, Liu PK (1999) Suppression of postischemic hippocampal 
nerve growth factor expression by a c-fos antisense oligodeoxynucleotide. J Neurosci 19:1335-1344.

de Marco G, Bogdanov A, Marecos E, Moore A, Simonova M, Weissleder R (1998) MR imaging of gene delivery to the central nervous system with an artificial vector. Radiology 208:65-71.

Enochs WS, Schaffer B, Bhide PG, Nossiff N, Papisov M, Bogdanov Jr A, Brady TJ, Weissleder R (1993) MR imaging of slow axonal transport in vivo. Exp Neurol 123:235-242.

Filler AG (1994) Axonal transport and MR imaging: prospects for contrast agent development. J Magn Reson Imaging 4:259-267.

Gass P, Fleischmann A, Hvalby O, Jensen V, Zacher C, Strekalova T, Kvello A, Wagner EF, Sprengel R (2004) Mice with a fra-1 knock-in into the c-fos locus show impaired spatial but regular contextual learning and normal LTP. Brain Res Mol Brain Res 130:16-22.

Hommel JD, Sears RM, Georgescu D, Simmons DL, DiLeone RJ (2003) Local gene knockdown in the brain using viral-mediated RNA interference. Nat Med 9:1539-1544.

Hope B, Kosofsky B, Hyman SE, Nestler EJ (1992) Regulation of immediate early gene expression and AP-1 binding in the rat nucleus accumbens by chronic cocaine. Proc Natl Acad Sci USA 89:5764-5768.

Hyman SE, Malenka RC (2001) Addiction and the brain: the neurobiology of compulsion and its persistence. Nat Rev Neurosci 2:695-703.

Kabnick KS, Housman DE (1988) Determinants that contribute to cytoplasmic stability of human c-fos and beta-globin mRNAs are located at several sites in each mRNA. Mol Cell Biol 8:3244-3250.

Kaczmarek L, Lapinska-Dzwonek J, Szymczak S (2002) Matrix metalloproteinases in the adult brain physiology: a link between c-Fos, AP-1 and remodeling of neuronal connections. EMBO J 21:6643-6648.

Kiyatkin EA (2004) Brain hyperthermia during physiological and pathological conditions: causes, mechanisms, and functional implications. Curr Neurovasc Res 1:77-90.

Konradi C, Cole RL, Heckers S, Hyman SE (1994) Amphetamine regulates gene expression in rat striatum via transcription factor CREB. J Neurosci 14:5623-5634.

Li YT, Liu HS, Lin HP, Chen SH (2005) Gold nanoparticles for microfluidics-based biosensing of PCR products by hybridizationinduced fluorescence quenching. Electrophoresis 26:4743-4750.

Lind K, Kresse M, Debus NP, Muller RH (2002) A novel formulation for superparamagnetic iron oxide (SPIO) particles enhancing MR lymphography: comparison of physicochemical properties and the in vivo behaviour. J Drug Target 10:221-230.

Liu CH, D'Arceuil HE, de Crespigny AJ (2004) Direct CSF injection of $\mathrm{MnCl}_{2}$ for dynamic manganese-enhanced MRI. Magn Reson Med 51:978-987.

Liu CH, Huang S, Kim YR, Rosen BR, Liu PK (2006) Forebrain ischemiareperfusion simulating cardiac arrest in mice induces edema and DNA fragmentation in the brain. Mol Imaging, in press.

Liu PK, Salminen A, He YY, Jiang MH, Xue JJ, Liu JS, Hsu CY (1994) Suppression of ischemia-induced fos expression and AP-1 activity by an antisense oligodeoxynucleotide to c-fos mRNA. Ann Neurol 36:566-576.

Mandeville JB, Marota JJ, Kosofsky BE, Keltner JR, Weissleder R, Rosen BR, Weisskoff RM (1998) Dynamic functional imaging of relative cerebral blood volume during rat forepaw stimulation. Magn Reson Med 39:615-624.

Mandeville JB, Jenkins BG, Kosofsky BE, Moskowitz MA, Rosen BR, Marota JJ (2001) Regional sensitivity and coupling of BOLD and CBV changes during stimulation of rat brain. Magn Reson Med 45:443-447.

Marshall JJ, Rabinowitz ML (1976) Preparation and characterization of a dextran-trypsin conjugate. J Biol Chem 251:1081-1087.

Moratalla R, Xu M, Tonegawa S, Graybiel AM (1996) Cellular responses to psychomotor stimulant and neuroleptic drugs are abnormal in mice lacking the D1 dopamine receptor. Proc Natl Acad Sci USA 93:14928-14933.

Morrow BA, Elsworth JD, Inglis FM, Roth RH (1999) An antisense oligonucleotide reverses the footshock-induced expression of fos in the rat medial prefrontal cortex and the subsequent expression of conditioned fear-induced immobility. J Neurosci 19:5666-5673.

Muldoon LL, Varallyay P, Kraemer DF, Kiwic G, Pinkston K, WalkerRosenfeld SL, Neuwelt EA (2004) Trafficking of superparamagnetic iron oxide particles (Combidex) from brain to lymph nodes in the rat. Neuropathol Appl Neurobiol 30:70-79.
Murphy CB, Zhang Y, Troxler T, Ferry V, Martin JJ, Jones Jr WE (2004) Probing Förster and Dexter energy-transfer mechanisms in fluorescent conjugated polymer chemosensors. J Phys Chem B 108:1537-1543.

Ogawa S, Brown HE, Okano HJ, Pfaff DW (1995) Cellular uptake of intracerebrally administered oligodeoxynucleotides in mouse brain. Regul Pept 59:143-149.

Parelkar NK, Wang JQ (2004) mGluR5-dependent increases in immediate early gene expression in the rat striatum following acute administration of amphetamine. Brain Res Mol Brain Res 122:151-157.

Paxinos G, Franklin KBJ (2001) The mouse brain in stereotoxic coordinates. London: Academic.

Perez JM, Josephson L, O'Loughlin T, Hogemann D, Weissleder R (2002) Magnetic relaxation switches capable of sensing molecular interactions. Nat Biotechnol 20:816-820.

Petropoulos AE, Schaffer BK, Cheney ML, Enochs S, Zimmer C, Weissleder R (1995) MR imaging of neuronal transport in the guinea pig facial nerve: initial findings. Acta Otolaryngol 115:512-516.

Raivich G, Behrens A (2006) Role of the AP-1 transcription factor c-Jun in developing, adult and injured brain. Prog Neurobiol 78:347-363.

Reynolds F, O'Loughlin T, Weissleder R, Josephson L (2005) Method of determining nanoparticle core weight. Anal Chem 77:814-817.

Ricart K, Pearson Jr RJ, Viera L, Cassina P, Kamaid A, Carroll SL, Estevez AG (2006) Interactions between beta-neuregulin and neurotrophins in motor neuron apoptosis. J Neurochem 97:222-233.

Roberts TP, Chuang N, Roberts HC (2000) Neuroimaging: do we really need new contrast agents for MRI. Eur J Radiol 34:166-178.

Sagar SM, Sharp FR, Curran T (1988) Expression of c-fos protein in brain: metabolic mapping at the cellular level. Science 240:1328-1331.

Saini S, Sharma R, Baron RL, Turner DA, Ros PR, Hahn PF, Small WC, Delange EE, Stillman AE, Edelman RR, Runge VM, Outwater EK (2000) Multicentre dose-ranging study on the efficacy of USPIO ferumoxtran-10 for liver MR imaging. Clin Radiol 55:690-695.

Sandberg DI, Edgar MA, Souweidane MM (2002) Convection-enhanced delivery into the rat brainstem. J Neurosurg 96:885-891.

Schedlich L, Hill M, Lockett T (1997) Antisense inhibition of beta-actin mRNA localization and its effect on smooth muscle cell migration. Biol Cell 89:113-122.

Schmauss C (2000) A single dose of methamphetamine leads to a long term reversal of the blunted dopamine D1 receptor-mediated neocortical c-fos responses in mice deficient for D2 and D3 receptors. J Biol Chem 275:38944-38948.

Shen T, Weissleder R, Papisov M, Bogdanov Jr A, Brady TJ (1993) Monocrystalline iron oxide nanocompounds (MION): physicochemical properties. Magn Reson Med 29:599-604.

Tolliver BK, Sganga MW, Sharp FR (2000) Suppression of c-fos induction in the nucleus accumbens prevents acquisition but not expression of morphine-conditioned place preference. Eur J Neurosci 12:3399-3406.

van Everdingen KJ, Enochs WS, Bhide PG, Nossiff N, Papisov M, Bogdanov Jr A, Brady TJ, Weissleder R (1994) Determinants of in vivo MR imaging of slow axonal transport. Radiology 193:485-491.

VanLandingham KE, Heinz ER, Cavazos JE, Lewis DV (1998) Magnetic resonance imaging evidence of hippocampal injury after prolonged focal febrile convulsions. Ann Neurol 43:413-426.

Wahlestedt C, Golanov E, Yamamoto S, Yee F, Ericson H, Yoo H, Inturrisi CE, Reis DJ (1993) Antisense oligodeoxynucleotides to NMDA-R1 receptor channel protect cortical neurons from excitotoxicity and reduce focal ischaemic infarctions. Nature 363:260-263.

Zhang M, Miller C, He Y, Martel-Pelletier J, Pelletier JP, Di Battista JA (1999) Calphostin C induces AP1 synthesis and AP1-dependent c-jun transactivation in normal human chondrocytes independent of protein kinase C-alpha inhibition: possible role for c-jun N-terminal kinase. J Cell Biochem 76:290-302.

Zhang Y, Widmayer MA, Zhang B, Cui JK, Baskin DS (1999) Suppression of post-ischemic-induced fos protein expression by an antisense oligonucleotide to $c$-fos mRNA leads to increased tissue damage. Brain Res 832:112-117.

Zhang YM, Wang Y, Liu N, Zhu ZH, Rusckowski M, Hnatowich DJ (2001) In vitro investigations of tumor targeting with $(99 \mathrm{~m}) \mathrm{Tc}$-labeled antisense DNA. J Nucl Med 42:1660-1669. 